\title{
Dielectric black holes induced by a refractive index perturbation and the Hawking effect.
}

\author{
F. Belgiorno ${ }^{1}$, S.L. Cacciatori ${ }^{2,3}$, G. Ortenzi ${ }^{4}$, L. Rizzi ${ }^{2}$, V. Gorini ${ }^{2,3}$, D. Faccio ${ }^{5}$ \\ 1 Dipartimento di Fisica, Università di Milano, Via Celoria 16, IT-20133 Milano, Italy \\ 2 Department of Physics and Mathematics, Università dell'Insubria, Via Valleggio 11, IT-22100 Como, Italy \\ ${ }^{3}$ INFN sezione di Milano, via Celoria 16, IT-20133 Milano, Italy \\ 4 Dipartimento di Matematica e Applicazioni, Università di Milano-Bicocca, Via Cozzi 53, IT-20125 Milano, Italy \\ 5 School of Engineering and Physical Sciences, SUPA Heriot-Watt University, Edinburgh, Scotland EH14 4AS, UK
}

(Dated: November 3, 2018)

\begin{abstract}
We consider a 4D model for photon production induced by a refractive index perturbation in a dielectric medium. We show that, in this model, we can infer the presence of a Hawking type effect. This prediction shows up both in the analogue Hawking framework, which is implemented in the pulse frame and relies on the peculiar properties of the effective geometry in which quantum fields propagate, as well as in the laboratory frame, through standard quantum field theory calculations. Effects of optical dispersion are also taken into account, and are shown to provide a limited energy bandwidth for the emission of Hawking radiation.
\end{abstract}

\section{INTRODUCTION}

One of the most intriguing predictions of quantum fields in curved spacetime geometries is the production of Hawking radiation. In 1974 S. Hawking predicted that black holes emit particles with a thermal spectrum. Therefore a black hole will evaporate, shedding energy under the form of a blackbody emission [1 3]. However, it turns out that for a typical stellar mass black hole the temperature of this radiation is so low $(\sim 10 \mathrm{nK})$ that it has no hope of being directly detected. Notwithstanding, Hawking radiation does not actually require a true gravitational event horizon but, rather, it is essentially a kinematical effect, i.e. it requires some basic "kinematical" ingredients but no specific underlying dynamics (see e.g. (4-6]). What suffices is the formation of a trapping horizon in a curved spacetime metric of any kind, and the analysis of the behavior of any quantum field therein (see also the discussion in [6]). The quanta of this field will then be excited according to the prediction of Hawking. On this basis, a number of analogue systems have been proposed, for the first time by W. Unruh [7] and later by other researchers (see e.g. 8 and references therein), that aim at reproducing some aspects of the kinematics of gravitational systems. Most of these analogies rely on acoustic perturbations and on the realization of so-called dumb holes: a liquid or gas medium is made to flow faster than the velocity of acoustic waves in the same medium so that at the transition point between sub and supersonic flow, a trapping horizon is formed that may be traversed by the acoustic quanta, viz. phonons, only in one direction [8. Unfortunately the phonon blackbody spectrum is expected to still have very low temperatures, thus eluding direct detection (see e.g. 9]). A parallel line of investigation involves effective geometry for light, which has been introduced by Gordon 10 and extended also to nonlinear electrodynamics; black hole metrics have been introduced and the possibility to perform experiments involving Hawking analogue radiation has been explored 112 23. Some time ago Philbin et al. proposed an optical analogue in which a soliton with intensity $I$, propagating in an optical fibre, generates through the nonlinear Kerr effect a refractive index perturbation (RIP), $\delta n=n_{2} I$, where $n_{2}$ is the Kerr index [24]. The same mechanism has also been generalized to a full $4 \mathrm{D}$ geometry by Faccio et al. 25] and has led to the first experimental observation of quanta emitted from an analogue horizon 26. The RIP modifies the spacetime geometry as seen by co-propagating light rays and, similarly to the acoustic analogy, if the RIP is locally superluminal, i.e. if it locally travels faster than the phase velocity of light in the medium, an horizon is formed and Hawking radiation is to be expected.

Here we take into consideration the Hawking effect in dielectric black holes induced by a RIP which propagates with constant velocity $v$. We first show that, under suitable conditions, we can re-map the original Maxwell equations for nonlinear optics into a geometrical description, in analogy to what is done in the case of acoustic perturbations in condensed matter. Then we provide a model in which the presence of the Hawking radiation can be inferred even without recourse to the analogous model characterized by a curved spacetime geometry. Indeed, Hawking radiation is a new phenomenon for nonlinear optics, never foreseen before, which could legitimately meet a sceptical attitude by the nonlinear optics community, because of a missing description of the phenomenon by means of the standard tools of quantum electrodynamics. As a consequence, it is important to recast the analogue picture by using "standard" tools of quantum field theory (without any reference to the geometrical picture).

The peculiar feature which distinguishes the dielectric Hawking effect from the traditional black hole one is the presence of optical dispersion, which gives rise to relevant physical consequences on the quantum phenomenon of particle creation. Therefore, we take dispersion into account. We first introduce a $2 \mathrm{D}$ reduction of the model in the presence of dispersion, and see how the dispersion 
relation is affected by the frequency-dependence of the refractive index. Then we discuss both phase velocity horizons and group velocity horizons, which can both play a relevant role in the physical situation at hand; their qualitative difference and the possibility to discriminate between them in experiments (numerical and/or laboratory-based) is considered.

The structure of the paper is as follows. In section II we introduce our idealized model of a RIP propagating, in a nonlinear Kerr medium, with a constant velocity $v$ in the $x$ direction and infinitely extended in the transverse $y$ and $z$ directions. In the eikonal approximation, the model is embodied in a suitable wave equation for the generic component of the electric field propagating in the nonlinear medium affected by the RIP. We describe this propagation as taking place in an analogue spacetime metric, written in the pulse frame, where the metric is static and displays two horizons $x_{+}$and $x_{-}$ for propagating photons, analogues to a black hole and a white hole horizons respectively. By assigning to the black hole horizon a surface gravity in a standard way, we calculate the evaporation temperature $T_{+}$of the RIP in the latter frame, which turns out to be proportional to the absolute value of the derivative of the RIP's profile, evaluated at $x_{+}$. We also show that $T_{+}$is conformally invariant, as expected for the consistency of the model.

In section III] we tackle the model from a different perspective, namely within the framework of quantum field theory. We construct a complete set of positive frequency solutions $\Phi_{\mathbf{k}_{l}}$ of the wave equation in the lab frame, in terms of which we quantize the field in the standard way. Then, we compare the $\Phi_{\mathbf{k}_{l}}$ with the corresponding plane wave solutions of the wave equation in the absence of the RIP. This enables us to calculate the Bogoliubov coefficients, in terms of which we express the expectation value of the number operator of the outgoing quanta in the in vacuum state. As expected, this average is a thermal-like distribution displaying the typical $\cos \theta$ temperature profile in the lab frame, where $\theta$ is the emission angle with respect to the direction of motion of the RIP. We then display the transformation law which relates the temperatures in the pulse and in the lab frame, respectively, by means of the usual Doppler formula.

The results worked out in the preceding sections apply in the approximation in which dispersion is neglected. In section [V] we study the modifications we expect when dispersion is taken into account. In particular, we show that, whereas in the dispersionless case one should expect to observe all the blackbody spectrum, in the presence of dispersion only a limited spectral region, which depends on $v$, will be excited, and the blackbody spectrum shape will not be discernible. Moreover, one can introduce two different concepts of horizon: phase velocity horizons and group velocity horizons, which are shown to be involved with different spectral bandwidths, and with very different qualitative behavior regarding their action on photons.
Section $\mathrm{V}$ is devoted to the conclusions.

Finally, we have added three appendices. The first establishes a correspondence between our black hole metric and the acoustic one. The second one relates the temperature to the standard conical singularity of the Euclidean version of the metric. The third establishes the relevant analytic properties of the Bogoliubov coefficients.

\section{STATIC DIELECTRIC BLACK HOLE}

We consider a model-equation which is derived from nonlinear electrodynamics (with $\chi_{2}=0$ and $\chi_{3} \neq 0$ ) in the eikonal approximation for a perturbation of a fullnonlinear electric field propagating in a nonlinear Kerr medium. In order to make the forthcoming analysis as simple as possible we replace the electric field with a scalar field $\Phi$ (cf. e.g. Schwinger's analysis of sonoluminescence 28]), for which we obtain the wave equation 29 .

$$
\frac{n^{2}\left(x_{l}-v t_{l}\right)}{c^{2}} \partial_{t_{l}}^{2} \Phi-\partial_{x_{l}}^{2} \Phi-\partial_{y}^{2} \Phi-\partial_{z}^{2} \Phi=0 .
$$

Coordinates in the lab frame are denoted by $t_{l}, x_{l}, y, z$ (the labels of $y, z$ are omitted because they will not be involved in the boost relating the lab frame with the pulse frame). Here, $n\left(x_{l}-v t_{l}\right)$ is the refractive index, which accounts for the propagating RIP in the dielectric. The latter can be obtained by means of an intense laser pulse in a nonlinear dielectric medium (Kerr effect). In our model the RIP does not depend on the transverse coordinates, namely it is infinitely extended in the transverse dimensions. This approximation is necessary in order to carry out the calculations below, which allow us to draw a tight analogy between the Hawking geometrical description and the quantum field theoretical treatment. Such an infinitely extended RIP is clearly an idealization, which cannot be obtained in an actual experiment. However, relatively flat, e.g. super-Gaussian-like pulses may be produced that would, at least locally, fall within the approximations adopted here.

Eq. (1) arises also in the eikonal approximation for a scalar field in the metric

$$
d s^{2}=\frac{c^{2}}{n^{2}\left(x_{l}-v t_{l}\right)} d t_{l}^{2}-d x_{l}^{2}-d y^{2}-d z^{2} .
$$

To carry out the analysis in the context of the analogue metric, we pass from the laboratory frame to the pulse frame (refractive index perturbation frame) by means of a boost: $t=\gamma\left(t_{l}-\frac{v}{c^{2}} x_{l}\right), x=\gamma\left(x_{l}-v t_{l}\right)$, and we obtain

$$
\begin{aligned}
d s^{2} & =c^{2} \gamma^{2} \frac{1}{n^{2}}\left(1+\frac{n v}{c}\right)\left(1-\frac{n v}{c}\right) d t^{2}+ \\
+2 \gamma^{2} \frac{v}{n^{2}}\left(1-n^{2}\right) d t d x-\gamma^{2}\left(1+\frac{v}{n c}\right)\left(1-\frac{v}{n c}\right) d x^{2}- & -d y^{2}-d z^{2}
\end{aligned}
$$




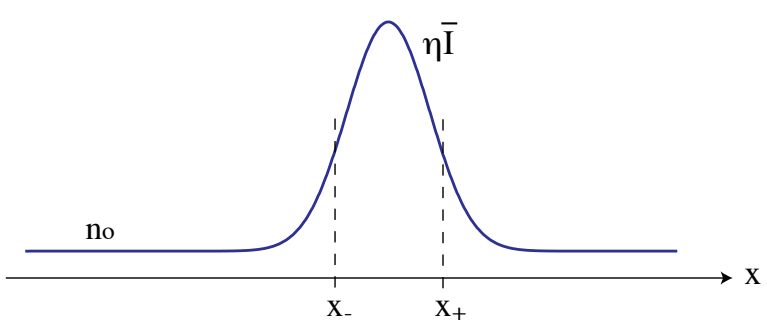

FIG. 1: (in color online) Scheme of the RIP geometry. $x_{+}$and $x_{-}$indicate the black hole and white hole horizon positions, respectively.

We assume

$$
n(x)=n_{0}+\delta n=n_{0}+\eta \bar{I}(x),
$$

where $\eta$ is meant to be positive, with $\eta \ll 1$ due to the actual smallness of the Kerr index (a negative $\eta$ could be easily taken into account); $\bar{I}$ denotes the normalized intensity of the pulse, is taken to be a $C^{\infty}$ function, rapidly decaying at infinity and with a single maximum at $x=0$, of height 1. A scheme of the RIP is shown in Fig. 1. The form of $\bar{I}$ implies that both $\partial_{t}=: \xi$ and $\partial_{\phi}$ are Killing vectors for the given metric. Then the surface $g_{00}=0$ is lightlike and corresponds to an event horizon. It is also possible to verify that the Frobenius integrability conditions are trivially satisfied, so that there exists a coordinate transformation carrying the metric into a static form (see e.g. [30]). Even if these coordinates are singular, we carry out the relative transformation because, on one hand, it allows a more straightforward comparison with the well-known Schwarzschild case, and on the other hand, it allows a direct computation of the greybody coefficient (cf. sect. IIID). To implement this transformation consider the following coordinate change:

$$
d t=d \tau-\alpha(x) d x
$$

where

$$
\alpha(x)=\frac{g_{01}(x)}{g_{00}(x)} .
$$

Then the metric takes the static form

$$
d s^{2}=\frac{c^{2}}{n^{2}(x)} g_{\tau \tau}(x) d \tau^{2}-\frac{1}{g_{\tau \tau}(x)} d x^{2}-d y^{2}-d z^{2},
$$

where

$$
g_{\tau \tau}(x):=\gamma^{2}\left(1+n(x) \frac{v}{c}\right)\left(1-n(x) \frac{v}{c}\right) .
$$

There is a remarkable resemblance of the $\tau, x$-part of the metric with a standard static spherically symmetric metric in general relativity in the so-called Schwarzschild gauge, aside from the important difference represented by the factor $\frac{c^{2}}{n^{2}}$ replacing $c^{2}$. The horizons are determined by the condition $g_{\tau \tau}=0$, i.e. by

$$
1-n(x) \frac{v}{c}=0
$$

which, by Eq. (4), becomes $n_{0}+\eta \bar{I}(x)=c / v$. Due to the specific shape assumed for $\bar{I}$ there will be two horizons: one, denoted by $x_{+}$, located on the rising edge of the RIP (i.e. $d n /\left.d x\right|_{x_{+}}<0$ ) and one, denoted by $x_{-}$, on the falling edge (i.e. $d n /\left.d x\right|_{x_{-}}>0$ ). Since $0 \leq \bar{I} \leq 1$, the condition for the occurrence of the event horizons is

$$
\frac{1}{n_{0}+\eta} \leq \frac{v}{c}<\frac{1}{n_{0}}
$$

The external region corresponds to $x<x_{-}$and to $x>x_{+}$. The leading horizon $x=x_{+}$is a black hole horizon, whereas the trailing one $x=x_{-}$is a white hole horizon. This can be understood as follows. Consider the front observer, i.e. the observer toward which the pulse is moving. Any photon starting from the region behind the front zero $x_{+}$cannot reach this observer before the latter is reached by the pulse. Indeed, the pulse is moving superluminally relative to any photon travelling in the region between the two zeros $x_{-}$and $x_{+}$. Therefore, the "leading" zero $x_{+}$corresponds to a black hole horizon. Analogously, the "trailing" zero $x_{-}$corresponds to a white hole horizon. See also Appendix A for further details.

\section{A. Surface gravity and temperature}

We can formally assign a temperature to the "black hole" horizon $x_{+}$by defining it in the usual way in terms of a "surface gravity" $\kappa_{+}$associated to the latter. We have

$$
\begin{aligned}
\kappa_{+}^{2} & :=-\left.c^{4} \frac{1}{2} g^{a b} g^{c d}\left(\nabla_{a} \xi_{c}\right)\left(\nabla_{b} \xi_{d}\right)\right|_{x=x_{+}}= \\
& -\frac{c^{4}}{2}\left[\frac{-2 \gamma^{4}}{n^{4}}\left(\frac{d n}{d x}\right)^{2}\right]_{x=x_{+}},
\end{aligned}
$$

or 51

$$
\kappa_{+}=\gamma^{2} v^{2}\left|\frac{d n}{d x}\left(x_{+}\right)\right| .
$$

Then, the temperature is given by the familiar formula which has been also derived in 24]:

$$
T_{+}=\frac{\kappa_{+} \hbar}{2 \pi k_{b} c}=\gamma^{2} v^{2} \frac{\hbar}{2 \pi k_{b} c}\left|\frac{d n}{d x}\left(x_{+}\right)\right| .
$$

We can arrive at formula 12 by several different methods. For the sake of completeness, in Appendix B we employ a different method to derive the expression (13) for the temperature, and its invariance with respect to the conformal factor. The latter aspect is well-known in General Relativity [31, and also in the frame of acoustic black holes 32 .

Since

$$
\frac{c}{v}=n\left(x_{+}\right)=n_{0}+k \eta
$$


with $k=\bar{I}\left(x_{+}\right) \in(0,1)$, we can rewrite equations 12 and 13 respectively as follows:

$$
\begin{gathered}
\kappa_{+}=\frac{c^{2}}{\left(n_{0}+k \eta\right)^{2}-1}\left|\frac{d n}{d x}\left(x_{+}\right)\right|, \\
T_{+}=\frac{\hbar c}{2 \pi k_{b}} \frac{1}{\left(n_{0}+k \eta\right)^{2}-1}\left|\frac{d n}{d x}\left(x_{+}\right)\right| .
\end{gathered}
$$

\section{B. Gaussian pulse}

As an example of a refractive index perturbation $\eta \bar{I}(x)$ consider a Gaussian normalized intensity of the pulse,

$$
\bar{I}(x)=\exp \left(-\frac{x^{2}}{2 \sigma^{2}}\right)
$$

and $\eta$ a small parameter. These choice reflects a typical situation in experimental optics. Then the horizons given by Eq. (9) ) occur for

$$
x_{ \pm}= \pm \sigma \sqrt{-2 \log \left[\left(\frac{c}{v}-n_{0}\right) \frac{1}{\eta}\right]}
$$

and $x_{-}=-x_{+}$. The surface gravity and the temperature are respectively given by

$$
\kappa_{+}=\gamma^{2} v^{2} \frac{k \eta}{\sigma} \sqrt{2 \log \frac{1}{k}}
$$

and by

$$
T_{+}=\frac{\kappa_{+} \hbar}{2 \pi k_{b} c} \sim \frac{\hbar c}{2 \pi k_{b}} \frac{1}{n_{0}^{2}-1} \frac{k \eta}{\sigma} \sqrt{2 \log \frac{1}{k}} .
$$

Typical values of the parameters are: $\sigma \sim 10^{-5} \mathrm{~m}, \eta \sim$ $10^{-3}, n_{0} \sim 1.45$. Then, for $k=\frac{1}{2}$, say, we get

$$
T_{+} \sim 2 \cdot 10^{-2} K
$$

which is greater than in standard cases of sonic black holes. This temperature value is not to be meant as characteristic, somewhat larger values may be obtained. See the following subsection.

Note that this is the temperature in the frame of the pulse, with which the geometry is associated. A further transformation is needed in order to recover the temperature in the laboratory frame (see the following section). Note also that the temperature is proportional to the derivative with respect to $x$ of the refractive index, evaluated at the black hole horizon. This is in agreement with the results of Ref. 24].

\section{Shockwave model}

Let us assume that the rear part (trailing edge) of the filament is characterized by a shockwave profile (see e.g.
[24]). This is a typical e.g. of spontaneous laser pulse dynamics in transparent media with third order nonlinearity. An analogous behavior is expected for the refractive index. As a consequence, in our model it is necessary to introduce, beyond the scale $2 \sigma$, which, roughly speaking, represents the overall spatial extension of the RIP, at least a further scale that corresponds to the 'thickness' of the region where the refractive index undergoes its most rapid variation. Then we can adopt a profile which is analytically described as follows: let us define

$$
H(x):=1+\tanh \left(\frac{\sigma+x}{\delta_{w h}}\right) \tanh \left(\frac{\sigma-x}{\delta_{b h}}\right),
$$

where $\delta_{w h}, \delta_{b h}>0$ are length scales describing the 'thikness' of the region over which a rapid variation of the refractive index occurs, and $2 \sigma>0$ corresponds to the overall spatial extension of the RIP. Compare also the choice of the velocity profile for acoustic geometries chosen in [33. Then for the refractive index profile we choose

$$
n(x)=n_{0}+\eta \frac{H(x)}{\max _{x} H(x)},
$$

It is evident that asymptotically $n$ converges to $n_{0}$ and the RIP is correctly normalized. This model can be also useful in view of exploring the possibility to obtain a bh-wh laser 33 .

From a physical point of view, we point out that, in the presence of a shock front typical values of $\delta_{w h}, \eta$ may be order of $\delta_{w h} \sim 10^{-6} \mathrm{~m}$ and $\eta \sim 5 \cdot 10^{-3}$ respectively, leading to $T_{+} \sim 1 \mathrm{~K}$.

\section{QUANTUM FIELD THEORETICAL TREATMENT: HAWKING RADIATION IN THE LABORATORY FRAME}

The deduction of Hawking radiation emission by a (non-extremal) black hole can also be carried out in a dynamical situation where the effects of the onset of a black hole horizon on quantum field theory modes is taken into account. See e.g. the seminal paper by Hawking [2] and also 27, where the equivalence between the static and the dynamical picture for Hawking radiation is considered. In the following section, we develop a dynamical picture for particle production, with the aim of not relying only on the analogue gravity picture but also obtaining the phenomenon in a non geometrical setting by means of standard tools of quantum field theory.

Consider a massless scalar field propagating in a dielectric medium and satisfying Eq. (1). It is not difficult to show that this equation arises in the eikonal approximation for the components of the electric field perturbation in a nonlinear Kerr medium [12, 15. $n\left(x_{l}-v t_{l}\right)$ is the refractive index, which accounts for the propagating refractive index perturbation in the dielectric.

In order to develop the model we adopt the following strategy: a) we look first for a complete set of solutions 
of equation (1); b) then we employ these solutions to perform a comparison between an initial situation, consisting of an unperturbed dielectric without a laser pulse, with a uniform and constant refractive index $n_{0}$, and a final situation where a laser pulse inducing a superluminal RIP is instead present. This scenario is analogous to the original situation envisaged and treated by Hawking [2] in which one considers an initial spherically symmetric astrophysical object, with no particles present at infinity, followed by a collapsing phase leading eventually to an evaporating Schwarzschild black hole. In analogy with the original treatment by Hawking, our aim is to evaluate the mean value of the number of quanta (calculated by means of final creation and annihilation operators) on the initial vacuum state.

\section{A. Out states}

Recast equation (1) in terms of the new retarded and advanced variables, respectively $u=x_{l}-v t_{l}$ and $w=$ $x_{l}+v t_{l}$ :

$$
\begin{aligned}
& \frac{n^{2}(u) v^{2}}{c^{2}}\left(\partial_{u}^{2} \Phi+\partial_{w}^{2} \Phi-2 \partial_{u} \partial_{w} \Phi\right)- \\
& \quad-\left(\partial_{u}^{2} \Phi+\partial_{w}^{2} \Phi+2 \partial_{u} \partial_{w} \Phi\right)-\partial_{y}^{2} \Phi-\partial_{z}^{2} \Phi=0
\end{aligned}
$$

and look for monochromatic solutions of the form

$$
\Phi(u, w, y, z)=A(u) \mathrm{e}^{i k_{w} w+i k_{y} y+i k_{z} z} .
$$

Then, the ansatz of Eq. 25 leads to

$$
\begin{aligned}
A^{\prime \prime}(u)+2 i k_{w} & \frac{c^{2}+n^{2}(u) v^{2}}{c^{2}-n^{2}(u) v^{2}} A^{\prime}(u)- \\
& -\left(k_{w}^{2}+\frac{k_{y}^{2}+k_{z}^{2}}{1-n^{2}(u) \frac{v^{2}}{c^{2}}}\right) A(u)=0 .
\end{aligned}
$$

Assuming $n(u)$ to be analytic (see e.g.(17)) we see that the coefficients of $A^{\prime}(u)$ and of $A(u)$ have a first order pole at the roots of

$$
1-n(u) \frac{v}{c}=0
$$

Then, assuming $n$ to be of the form (4), the condition for the occurrence of the event horizons is given by 10 . Since $x=\gamma u$, the black hole and white hole horizons, when they exist, are located respectively at

$$
u_{ \pm}=\frac{1}{\gamma} x_{ \pm}
$$

and we have a second order linear differential equation with Fuchsian singular points at $u=u_{ \pm}$(with $u_{-}<u_{+}$). The general solution of Eq.224) can be determined, in the neighbourhood of the singular points $u_{ \pm}$, by the standard methods of integration by series. We perform this calculation in Appendix C Here, instead, since we are interested in the asymptotic behaviour of the solutions as $u \rightarrow u_{ \pm}$and as $u \rightarrow \pm \infty$, the best strategy is to appeal to the WKB approximation. Namely, we write $\Phi$ as in Eq. 25 with

$$
A(u)=\exp \left(i \int^{u} k_{u}\left(u^{\prime}\right) d u^{\prime}\right) .
$$

Then, we find the following dispersion relation:

$$
\frac{n^{2} v^{2}}{c^{2}}\left(k_{u}-k_{w}\right)^{2}-\left(k_{u}+k_{w}\right)^{2}-k_{\perp}^{2}=0
$$

where $k_{\perp}^{2}=k_{y}^{2}+k_{z}^{2}$. By solving for $k_{u}$ as a function of $k_{w}, \mathbf{k}_{\perp}$ one arrives at a second degree equation whose solutions are of the form:

$$
\begin{aligned}
k_{u}^{ \pm}= & -\frac{k_{w}}{1-\frac{n^{2} v^{2}}{c^{2}}}\left[1+\frac{n^{2} v^{2}}{c^{2}} \pm\right. \\
& \pm 2 \frac{n v}{c} \sqrt{\left.1-\frac{k_{\perp}^{2}}{k_{w}^{2}} \frac{1-\frac{n^{2} v^{2}}{c^{2}}}{4 \frac{n^{2} v^{2}}{c^{2}}}\right]} .
\end{aligned}
$$

The constraint

$$
\frac{k_{\perp}^{2}}{k_{w}^{2}} \leq \frac{4 \frac{n^{2} v^{2}}{c^{2}}}{1-\frac{n^{2} v^{2}}{c^{2}}}
$$

must hold in order that propagating solutions are available. This seemingly would imply the existence of a limiting angle for the emission, but one has to take into consideration that, in approaching the horizon, the right hand side of the latest equationn becomes infinite, leaving room for no real limitation on the emission angle.

The root $k_{u}^{+}$is singular at the horizons, whereas $k_{u}^{-}$is regular there. We focus on the behaviour of $k_{u}^{+}$in the neighbourhood of $u_{+}$. Near $u_{+}$the dependence on $\mathbf{k}_{\perp}$ is washed out, in agreement with the analysis in [5], and we have

$$
k_{u} \sim-\frac{2 k_{w}}{1-n \frac{v}{c}}
$$

Then,

$$
A(u) \sim \exp \left[i \frac{2 c k_{w}}{v n^{\prime}\left(u_{+}\right)} \log \left(u-u_{+}\right)\right] .
$$

The logarithmic divergence in the phase as $u \downarrow u_{+}$is entirely analogous to the one experienced by the covariant wave equation at the horizon of a Schwarzschild black hole [2, 3] and represents the typical behaviour of outgoing modes approaching the horizon [5]. It is worth to remark that this divergence appears here without any reference to geometry. In addition, as shown in Appendix C, it emerges naturally as an exact asymptotic (as $u \downarrow u_{+}$) consequence of Eq. 24. On the other hand, the same asymptotic behaviour can be inferred also in the context of the geometric approach. Indeed, such behaviour is characteristic also of the monochromatic solutions of the 
covariant wave equation in the metric (2), though the latter equation is not exactly solvable.

Regarding the dependence on $\vec{k}_{\perp}$, we note that it does not disappear far away from the horizon, but this turns out not to be relevant for the Hawking phenomenon [5]. Also note that $k_{u}$ has the opposite sign of $k_{w}$, and $\left|k_{u}\right|>$ $\left|k_{w}\right|$.

We denote by $\mathrm{F}^{+}$the solutions associated to the root $k_{u}^{+}$. Their asymptotic form (at large distances from the pulse, i.e. for $u \gg u_{+}$) is

$$
F^{+} \sim e^{i k_{u} u+i k_{w} w+i k_{y} y+i k_{z} z},
$$

or

$$
F^{+} \sim e^{i\left(k_{u}+k_{w}\right) x_{l}+i k_{y} y+i k_{z} z-i v t_{l}\left(k_{u}-k_{w}\right)} .
$$

This can be written as

$$
F^{+}=F_{\mathbf{k}_{l}}^{+} \sim \exp \left(i \mathbf{k}_{l} \cdot \mathbf{x}_{l}-i \omega_{l} t_{l}\right),
$$

where $\mathbf{k}_{l}=\left(k_{x l}, k_{y}, k_{z}\right)$, with

$$
\begin{aligned}
\omega_{l} & =v\left(k_{u}-k_{w}\right), \\
k_{x l} & =k_{u}+k_{w},
\end{aligned}
$$

so that

$$
\begin{aligned}
& k_{u}=\frac{1}{2}\left(k_{x l}+\frac{\omega_{l}}{v}\right), \\
& k_{w}=\frac{1}{2}\left(k_{x l}-\frac{\omega_{l}}{v}\right) .
\end{aligned}
$$

We look for solutions which are asymptotically of positive frequency and outgoing with respect to the dielectric perturbation, which is right moving. In other words, we choose $\omega_{l}>0$ and $k_{x l}>0$. Moreover, for definiteness we fix $k_{w}<0$. As a consequence, (38) and $(39)$ are satisfied for $k_{u}>-k_{w}$. As to $\omega_{l}$, it satisfies the asymptotic dispersion relation $n_{0}^{2} \omega_{l}^{2}=\mathbf{k}_{l}{ }^{2} c^{2}$. A similar analysis can be performed for the solution which is regular at $u=u_{+}$, which we denote by $F_{\mathbf{k}_{l}, r e g}^{+}$. Similarly, we denote by $F_{\mathbf{k}_{l}}^{-}$ and by $F_{\mathbf{k}_{l}, \text { reg }}^{-}$the singular and, respectively, regular solutions at $u_{-}$.

Introducing the angle of emission $\theta$, defined by $k_{x}=$ $\left|\mathbf{k}_{l}\right| \cos (\theta)$, Eq. 411 can be written as

$$
k_{w}=-\frac{\omega_{l}}{2 v}\left(1-\frac{v}{c} n_{0} \cos (\theta)\right) .
$$

\section{B. In states} is

Prior to the formation of the pulse the wave equation

$$
\frac{n_{0}^{2}}{c^{2}} \partial_{t_{l}}^{2} \Phi-\partial_{x_{l}}^{2} \Phi-\partial_{y}^{2} \Phi-\partial_{z}^{2} \Phi=0,
$$

whose independent positive frequency plane wave solutions are trivially given by (with $\omega_{l}>0$ ),

$$
F_{\mathbf{k}_{l}}^{\text {in }}=\exp \left(i \mathbf{k}_{l} \cdot \mathbf{x}_{l}-i \omega_{l} t_{l}\right),
$$

where the obvious dispersion relation holds:

$$
\frac{n_{0}^{2}}{c^{2}} \omega_{l}^{2}-k_{x l}^{2}-k_{\perp}^{2}=0 .
$$

and where $k_{\perp}^{2}=k_{y}^{2}+k_{z}^{2}$.

\section{Quantization}

We can separate the monochromatic solutions $\left\{F^{+}, F_{\text {reg }}^{+}\right\}$corresponding to all possible values of $\mathbf{k}_{l}$, into positive and negative frequency components $\left\{f_{\mathbf{k}_{l}}, f_{\mathbf{k}_{l}}^{\star}\right\}$. These form a complete set of solutions which are mutually orthogonal w.r.t. the index $\mathbf{k}_{l}$ relative to the KleinGordon product in the retarded variables:

$$
(\Psi, \Phi)=i \int_{\mathbb{R}^{3}} d u d y d z\left(\Psi^{*} \frac{\partial \Phi}{\partial w}-\Phi \frac{\partial \Psi^{*}}{\partial w}\right) .
$$

The above product is a function of $w$ (due to the factor $n^{2}\left(x_{l}-v t_{l}\right)$ it is indeed impossible to define in a standard way a conserved inner product for Eq. (10). Expanding an arbitrary solution $\Phi$ of Eq. (1) over the modes $\left\{f_{\mathbf{k}_{l}}, f_{\mathbf{k}_{l}}^{\star}\right\}$ we quantize the field by promoting $\Phi$ to a field operator according to the expansion

$$
\Phi=\int d^{3} \mathbf{k}_{l}\left(a_{\mathbf{k}_{l}} f_{\mathbf{k}_{l}}+a_{\mathbf{k}_{l}}^{\star} f_{\mathbf{k}_{l}}^{\star}\right),
$$

where the creation and annihilation operators $a_{\mathbf{k}_{l}}, a_{\mathbf{k}_{l}}^{\star}$ satisfy the usual commutation relations

$$
\left[a_{\mathbf{k}_{l}}, a_{\mathbf{k}_{l^{\prime}}}^{\star}\right]=\delta_{\mathbf{k}_{l} \mathbf{k}_{l^{\prime}}} .
$$

Comparison between the creation and annihilation operators of the in and out states will allow us to calculate the Bogoliubov coefficients relating one set to the other, thus leading us to evaluate the average number of emitted quanta.

\section{Greybody factor}

In our four dimensional problem, a priori we cannot neglect the effect of backscattering, i.e., the fact that, once emitted near the horizon, photons can undergo with a certain probability, reflection back in to the horizon due to the presence of a non-vanishing potential that they encounter in their propagation 2. This originates the so-called greybody factor, which can be calculated as the square modulus of the transmission coefficient.

Consider a massless scalar field propagating in a dielectric medium and satisfying the massless Klein-Gordon equation in the pulse metric (7), and let us call $g(x)$ the component $g_{\tau \tau}$ appearing in (8). We get

$$
\frac{n^{2}(x)}{c^{2}} \frac{1}{g(x)} \partial_{\tau}^{2} \Phi-n(x) \partial_{x}\left(\frac{1}{n(x)} g(x) \partial_{x} \Phi\right)-\partial_{y}^{2} \Phi-\partial_{z}^{2} \Phi=0 .
$$


We consider solutions of the form

$$
\Phi(\tau, x, y, z)=e^{i \omega \tau} \varphi(x) e^{-i k_{y} y-i k_{z} z} .
$$

As a consequence, we obtain an equation of the form

$$
\left(p(x) \varphi^{\prime}\right)^{\prime}+\omega^{2} k(x) \varphi-q(x) \varphi=0,
$$

where the prime stays for the derivative w.r.t. $x$ and

$$
p(x):=\frac{g}{n} ; \quad k(x):=\frac{n}{c^{2} g} ; \quad q(x):=k_{\perp}^{2} \frac{1}{n} .
$$

The following change of variable:

$$
s(x):=\int^{x} d u \sqrt{\frac{k(u)}{p(u)}}=\int^{x} d u \frac{n}{c g},
$$

where $s$ results to coincide with the tortoise coordinate, leads to the following Schrodinger-like form of the equation for $\varphi$ :

$$
\frac{d^{2} \varphi}{d s^{2}}+\left(\omega^{2}-Q(s)\right) \varphi=0
$$

where

$$
Q(s):=k_{\perp}^{2} \frac{c^{2} g}{n} .
$$

It can be noticed that, as $x \rightarrow x_{+}$, i.e. as $s \rightarrow-\infty$, one obtains $Q(s) \rightarrow 0$, and that

$$
\lim _{s \rightarrow \infty} Q(s)=k_{\perp}^{2} \frac{c^{2}}{n_{0}^{2}}\left(1-n_{0}^{2} \frac{v^{2}}{c^{2}}\right)=: Q_{\infty} .
$$

$Q(s)$ turns out to be monotonically increasing from 0 to the aforementioned constant value as $s \rightarrow \infty$, and the transition from the zero value and the asymptotically constant value for a bump is very fast, in such a way that, as a plausible approximation for the calculation of the transmission coefficient, we replace the above potential $Q(s)$ with a step-like effective potential with height $Q_{\infty}$ :

$$
\frac{d^{2} \varphi}{d s^{2}}+\left(\omega^{2}-Q_{\infty}\right) \varphi=0 .
$$

In this approximation, we can refer to well-known results of standard quantum mechanics (see e.g. 34, where the step barrier appears as a subcase of problem 37), in order to infer that:

a) the transmission coefficient is zero if

$$
\omega<\sqrt{Q_{\infty}}=k_{\perp} \frac{c}{n_{0}} \sqrt{\left(1-n_{0}^{2} \frac{v^{2}}{c^{2}}\right)} .
$$

b) the transmission coefficient is

$$
|T|^{2}=4 \frac{\omega \sqrt{Q_{\infty}}}{\left(\omega+\sqrt{Q_{\infty}}\right)^{2}}=\Gamma
$$

for

$$
\omega>\sqrt{Q_{\infty}}
$$

We point out that the dispersion relation

$$
k_{x}^{2}+k_{\perp}^{2}=\frac{n_{0}^{2}}{c^{2}} \omega^{2}
$$

implies that a) cannot occur. As a consequence, the Heaviside function which, in principle, should multiply the aforementioned $|T|^{2}$, is always equal to 1 .

We have to translate the latter result in the laboratory frame; the simple substitution

$$
\omega=\left(\omega_{l}-v k_{x l}\right) \gamma
$$

where the label $l$ indicates laboratory frame quantities, leads to the following expression for the greybody factor:

$$
\Gamma\left(\omega_{l}, \mathbf{k}_{\perp}\right) \simeq \frac{4\left(\omega_{l}-v k_{x l}\right) \sqrt{\left(\omega_{l}-v k_{x l}\right)^{2}-k_{\perp}^{2} q_{0}^{2}}}{\left[\left(\omega_{l}-v k_{x l}\right)+k_{\perp} q_{0}\right]^{2}}
$$

where

$$
q_{0}=\frac{c}{n_{0}} \sqrt{1-n_{0}^{2} \frac{v^{2}}{c^{2}}}
$$

One can also take into account that

$$
\omega_{l}-v k_{x l}=\omega_{l}\left(1-n_{0} \frac{v}{c} \cos (\theta)\right)
$$

so that

$$
\begin{aligned}
\Gamma & \simeq \frac{4\left(1-n_{0} \frac{v}{c} \cos (\theta)\right)\left|n_{0} \frac{v}{c}-\cos (\theta)\right|}{\left[1-n_{0} \frac{v}{c} \cos (\theta)+\left|n_{0} \frac{v}{c}-\cos (\theta)\right|\right]^{2}} \\
& \times \theta_{H}\left(\frac{\omega}{q_{0}}\left(1-n_{0} \frac{v}{c} \cos (\theta)\right)-k_{\perp}\right) .
\end{aligned}
$$

Being $\eta \ll 1$, and $v / c=\left(n_{0}+k \eta\right)^{-1}, k \in(0,1)$, we obtain

$$
\begin{aligned}
& 1-n_{0} \frac{v}{c} \cos (\theta) \sim 1-\cos (\theta)+\frac{k \eta}{n_{0}} \cos (\theta), \\
& n_{0} \frac{v}{c}-\cos (\theta) \sim 1-\cos (\theta)-\frac{k \eta}{n_{0}},
\end{aligned}
$$

which leads to the conclusion that

$$
\Gamma \simeq 1
$$

It is worth pointing out that, on the grounds of the previous result, a 90-degrees emission would not suffer any substantial suppression by backscattering. This is particularly relevant in connection to recent measurements of 90-degree photon emission from RIP-induced horizon 26. 


\section{E. Thermal spectrum}

In analogy with the corresponding black hole calculations, in order to find the evaporation temperature of the RIP we must compute the expectation value of the number operator of the outgoing photons evaluated in the in vacuum. In this connection, it is important to remark that, contrary to the standard black hole scenario, in the case of a RIP propagating in a nonlinear Kerr medium we have, strictly speaking, no real collapse situation. Nevertheless, we can "simulate a collapse" by building up a refractive index perturbation starting from an initial situation in which no signal is present in the dielectric.

We perform a coordinate shift which moves the black hole horizon in $u=0$. A photon starting at $u<0$ is trapped an cannot reach the front observer. As a consequence, states with $u<0$ cannot be available to the front observer and he will be lead to consider in his description only the $u>0$ part of the $F^{+}$solution, thus multiplying the latter by a Heaviside function $\theta(u)$. The Bogoliubov coefficients $\alpha_{\mathbf{k}_{l} \mathbf{k}_{l^{\prime}}}$ and $\beta_{\mathbf{k}_{l} \mathbf{k}_{l}{ }^{\prime}}$ relating, respectively, the positive and negative frequency components between the initial in and final out states are analysed in $\mathrm{D}$, and satisfy the fundamental relation

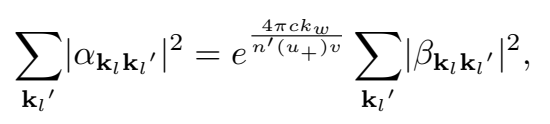

which is also proved in the appendix. Moreover, the expectation value of the number operator of the outgoing photons in the initial vacuum state is

$$
\left\langle 0 \text { in }\left|N_{\mathbf{k}_{l}}^{\text {out }}\right| 0 \text { in }\right\rangle=\sum_{\mathbf{k}_{l^{\prime}}}\left|\beta_{\mathbf{k}_{l} \mathbf{k}_{l}{ }^{\prime}}\right|^{2},
$$

Then, by using the completeness relation for the Bogoliubov coefficients and taking into account backscattering:

$$
\sum_{\mathbf{k}_{l^{\prime}}}\left(\left|\alpha_{\mathbf{k}_{l} \mathbf{k}_{l^{\prime}}}\right|^{2}-\left|\beta_{\mathbf{k}_{l} \mathbf{k}_{l}{ }^{\prime}}\right|^{2}\right)=\Gamma\left(\omega_{l}, \mathbf{k}_{\perp}\right)
$$

and Eq. (70), we obtain the following thermal-like distribution written in terms of asymptotic (physical) frequencies:

$$
\left\langle N_{\mathbf{k}_{l}}\right\rangle=\frac{\Gamma}{\exp \left(\frac{\hbar \omega_{l}}{k_{b} T}\right)-1}
$$

\section{EFFECTS OF OPTICAL DISPERSION}

In the framework of analogue models for black holes (dumb holes, bec models) there is a number of studies devoted to the analysis of the actual generation of Hawking radiation (see e.g. [9, 33, 35, 38, 40,43). In where

$$
T=v^{2} \frac{\hbar}{2 \pi k_{B} c} \frac{1}{1-\frac{v}{c} n_{0} \cos \theta}\left|\frac{d n}{d u}\left(u_{+}\right)\right| .
$$

By comparing this temperature with the temperature $T_{+}$ derived in the analogue Hawking model in the pulse frame (see Eq. 13), we find the relation:

$$
T=\frac{1}{\gamma} \frac{1}{1-\frac{v}{c} n_{0} \cos (\theta)} T_{+} .
$$

For the same values of the parameters leading to Eq. [21, for $\theta=0$ and for a typical value $v \sim \frac{2}{3} c$, we would obtain

$$
T \sim 78 K,
$$

which is again much greater than the values of $T$ for typical acoustic black holes. Moreover, in the specific case of a shock front, this temperature may increase significantly so that $T \sim 2000 K$ (cf. subsection IIC).

Equation. (75) gives the correct transformation law for the temperature in going over from the pulse frame to the laboratory frame. Indeed, to find how the temperature transforms, start from Wien's displacement law which gives the wavelength of maximum emission of a black body as a function of the temperature

$$
\lambda_{\max } T=b=2.9 \times 10^{-3} m \times K .
$$

Converting to the frequency of maximum emission gives

$$
\omega_{l \max }=\frac{2 \pi c}{b} T .
$$

Now, under the boost connecting the lab frame to the pulse frame the frequency transforms according to the relativistic Doppler formula in a medium with refractive index $n_{0}$

$$
\omega=\omega_{l} \gamma\left(1-\frac{v}{c} n_{0} \cos \theta\right),
$$

where $\omega$ is the frequency in the pulse frame, and $\theta$ is the emission angle with respect to the direction of motion of the pulse in the lab frame. Combining Eq. 790 with Eq. 78) gives Eq. 75).

particular, a suitable dispersion law, which involves the fourth power of the momentum $k$ in the comoving frame is considered, since the original model by Unruh [35. Calculations, in particular in presence of a black hole white hole system, are quite involved and require also numerical simulations. 


\begin{tabular}{|c|c|}
\hline Black Hole - Pulse Frame & Quantum Field Theory - Lab Frame \\
\hline $\begin{array}{c}\text { propagation equation (eikonal approximation) } \\
\downarrow \\
\text { appearance of a bh horizon } \\
\downarrow \\
\text { logarithmic singularity in the phase of quantum modes } \\
\downarrow \\
\text { thermal spectrum with temperature } T_{+}\end{array}$ & $\begin{array}{c}\text { propagation equation (eikonal approximation) } \\
\downarrow \\
\downarrow \\
\text { onset of the surface s.t. } 1-n(u) \frac{v}{c}=0 \\
\downarrow \\
\text { logarithmic singularity in the phase of quantum modes } \\
\downarrow \\
\text { thermal spectrum with temperature } T\end{array}$ \\
\hline
\end{tabular}

TABLE I: Comparison between the analogue Hawking and QFT descriptions of quantum vacuum emission by a moving RIP.

We do not deal herein quantitatively with the problem of mode conversion, and we do not consider the problem of black hole lasers (see 9, 33, 35, 43]), which will be taken into account in further developments of the present model. Still, we aim to point out the main physical consequences due to optical dispersion, and we shall stress that optical dispersion behaves as a fundamental ingredient for dielectric models. In this respect, there is agreement with dispersive models in sonic black holes.

We start by recalling how optical dispersion affects the dispersion relation for the quantum electromagnetic field in linear homogeneous dielectric media (see e.g. [44]):

$$
n^{2}\left(\omega_{l}\right) \omega_{l}^{2}-\mathbf{k}_{l}^{2} c^{2}=0 .
$$

This dispersion relation is obeyed by the monochromatic components of the field, and in 44] phenomenological quantization of the electromagnetic field in a dielectric medium is justified on the grounds of a more rigorous approach. In the presence of optical dispersion

$$
\Phi\left(\mathbf{x}_{l}, t_{l}\right)=\int_{0}^{\infty} d \omega_{l} \phi\left(\omega_{l}, \mathbf{x}_{l}, t_{l}\right)
$$

where $\phi\left(\omega_{l}, \mathbf{x}_{l}, t_{l}\right)=\varphi_{+}\left(\omega_{l}, \mathbf{x}_{l}\right) \mathrm{e}^{-i \omega_{l} t_{l}}+\varphi_{-}\left(\omega_{l}, \mathbf{x}_{l}\right) \mathrm{e}^{i \omega_{l} t_{l}}$ are the monochromatic components. Then it is easy to show that, even by allowing a spatial dependence for the refractive index

$$
\nabla \varphi\left(\omega_{l}, \mathbf{x}_{l}\right)+\omega_{l}^{2} \frac{n^{2}\left(\omega_{l}, \mathbf{x}_{l}\right)}{c^{2}} \varphi\left(\omega_{l}, \mathbf{x}_{l}\right)=0
$$

Moreover, in the case of an homogeneous medium, by letting $\varphi(\omega, \mathbf{x}) \propto \exp \left(i \mathbf{k}_{l} \cdot \mathbf{x}\right)$, one finds that eqn. 82 is satisfied only if

$$
\left|\mathbf{k}_{l}\right|=\omega_{l} \frac{n\left(\omega_{l}\right)}{c}
$$

i.e. only if each monochromatic component travels at its phase velocity (as obvious).
By keeping into account a so-called 1-resonance model, we use the Sellmeier equation

$$
n^{2}\left(\omega_{l}\right)=1+\frac{\omega_{c}^{2}}{\omega_{0}^{2}-\omega_{l}^{2}},
$$

where $\omega_{0}$ is the resonance frequency and $\omega_{c}$ is the coupling constant, which is also called plasma frequency. It is worth noting that the function $n^{2}\left(\omega_{l}\right)$ is a Lorentz scalar and it is invariant under boosts. (84) amounts to a quartic equation in $\omega_{l}$ :

$$
\omega_{l}^{4}-\left(\omega_{L}^{2}+\mathbf{k}_{l}^{2} c^{2}\right) \omega_{l}^{2}+\omega_{0}^{2} \mathbf{k}_{l}^{2} c^{2}=0,
$$

where $\omega_{L}^{2} \equiv \omega_{0}^{2}+\omega_{c}^{2}$ is the longitudinal resonance frequency. Solutions are

$$
\omega_{l \pm}^{2}=\frac{1}{2}\left[\omega_{L}^{2}+\mathbf{k}_{l}^{2} c^{2} \pm \sqrt{\left(\omega_{L}^{2}+\mathbf{k}_{l}^{2} c^{2}\right)^{2}-4 \omega_{0}^{2} \mathbf{k}_{l}^{2} c^{2}}\right]
$$

Then one finds two branches, which occur for $0 \leq \omega_{l-}<$ $\omega_{0}$ and $\omega_{L} \leq \omega_{l+}<\infty$ respectively, with a forbidden region in the interval $\left(\omega_{0}, \omega_{L}\right)$.

It is worth pointing out that, even if more complete expressions can be provided for the dispersion relation, for practical purposes it is very useful the so called Cauchy formula

$$
n\left(\omega_{l}\right)=n_{0}+B_{0} \omega_{l}^{2},
$$

where $n_{0}$ is the would-be refractive index in absence of optical dispersion and $B_{0}$ is a suitable constant; this approximation works well for fused silica in the visible frequency interval, and can also be obtained from the more complete formula 84 in the formal limit as $\omega_{l} \rightarrow 0$ (i.e., from a physical point of view, as $\left.\omega_{l} \ll \omega_{0}\right)$. For our aims, a posteriori, its limited bandwidth validity is not a real problem, because it turns out that also Hawking effect takes place in a limited frequency window.

\section{A. Dispersion and RIP}

We note that the dispersion relation for a linear homogeneous dielectric medium is quite different from the one 
which has been hitherto considered in the sonic model by Unruh and its subsequent developments (see also analogous comments in Ref. [45]).

We may also wonder if the above relation can be trusted in our case, as we started from a nonlinear medium which is also non-homogeneous because of the RIP. We recall that we assume to be involved with linearized quantum fields around our effective geometry, and then it is reasonable to explore what happens in presence of optical dispersion by considering linear dispersion effects. Moreover, we make the following ansatz:

$$
n\left(x_{l}-v t_{l}, \omega_{l}\right)=n_{0}\left(\omega_{l}\right)+\eta f\left(x_{l}-v t_{l}\right),
$$

i.e. we neglect optical dispersion effects in the RIP, and keep trace of it only in the background value $n_{0}$, which does not depend on spacetime variables, and passes from the status of constant to that of a function depending on $\omega_{l}$.

In order to justify a posteriori this ansatz we can take into account what happens in the usual modelization of dielectric media when inhomogeneities are considered. A possibility is to account for inhomogeneities by including space-dependent density, polarizability, resonant frequencies, and so on (see e.g. [46]). By neglecting dissipation in a polariton model, one can obtain a dielectric susceptibility which depends on space and frequency, in such a way that the Sellmeier formula changes only because of an explicit dependence of $\omega_{c}, \omega_{0}$ on space variables. In our case, because of the properties of fused silica in the visible region, where the Cauchy formula works well, we can even neglect the spatial dependence of $\omega_{0}$ (which should be considered much greater than $\omega_{l}$ and $\omega_{c}$ ), and then, recalling that $u:=x_{l}-v t_{l}$, on the grounds of Ref. [46] we assume that

$$
\omega_{c}^{2}(u)=\omega_{c 0}^{2}+\delta \omega_{c 1}^{2}(u),
$$

where $\delta \ll 1$ is a small parameter; if we also define

$$
n_{0}\left(\omega_{l}\right)=\sqrt{1+\frac{\omega_{c 0}^{2}}{\omega_{0}^{2}-\omega_{l}^{2}}},
$$

the Sellmeier equation 84 then leads to

$$
\begin{aligned}
n\left(\omega_{l}, u\right) & =n_{0}\left(\omega_{l}\right) \sqrt{1+\delta \frac{1}{n_{0}^{2}\left(\omega_{l}\right)} \frac{\omega_{c 1}^{2}(u)}{\omega_{0}^{2}-\omega_{l}^{2}}} \\
& \sim n_{0}\left(\omega_{l}\right)+\frac{\delta}{2 n_{0} \omega_{0}^{2}} \omega_{c 1}^{2}(u),
\end{aligned}
$$

where we have used the Cauchy approximation and neglected terms order of $\delta \omega_{l}^{2}$. This validates our ansatz (88), with straightforward identifications.

Another possibility is to adopt in the visible frequency region the following perturbative ansatz:

$$
\begin{aligned}
n\left(\omega_{l}, u\right) & =n_{0}+\eta A_{1}(u)+\left(B_{0}+\eta B_{1}(u)\right) \omega_{l}^{2} \\
& \sim n_{0}+B_{0} \omega_{l}^{2}+\eta A_{1}(u),
\end{aligned}
$$

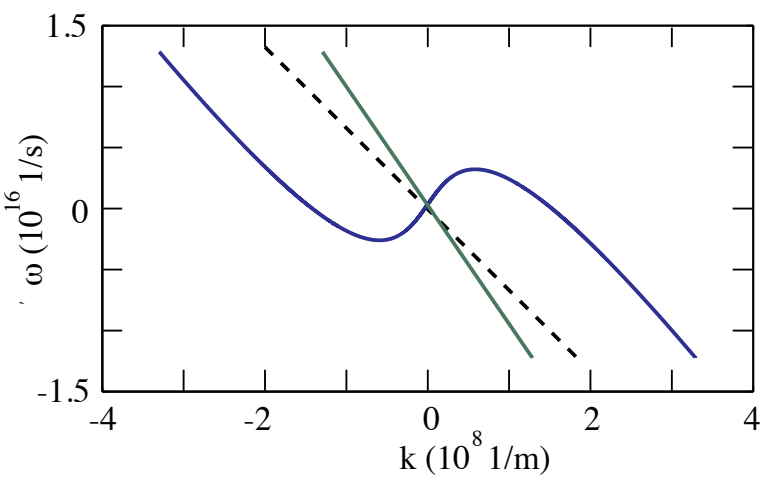

FIG. 2: (in color online) Branches relative to the dispersion relation $(96)$ in the comoving frame. The dashed line corresponds to $\omega=-v k_{x}$.

where $n_{0}+B_{0} \omega_{l}^{2} \equiv n_{0}\left(\omega_{l}\right)$ and where we neglect terms $O\left(\eta \omega_{l}^{2}\right)$. The latter approximation is very useful, because we can write

$$
n\left(\omega_{l}, u\right)=n_{0}\left(\omega_{l}\right)+\eta f(u)=n(u)+B_{0} \omega_{l}^{2},
$$

which isolates the contribution of the optical dispersion. As for the dispersion relation, we obtain in the lab

$$
n\left(\omega_{l}, u\right) \omega_{l}= \pm\left|\mathbf{k}_{l}\right| c
$$

i.e.

$$
n(u) \omega_{l}+B_{0} \omega_{l}^{3} \pm\left|\mathbf{k}_{l}\right| c=0 .
$$

This can be carried into the comoving (pulse) frame, where one obtains

$$
\begin{aligned}
& n(x) \gamma\left(\omega+v k_{x}\right) \pm \sqrt{\gamma^{2}\left(k_{x}+\frac{v}{c^{2}} \omega\right)^{2}+k_{\perp}^{2}} c \\
& +B_{0} \gamma^{3}\left(\omega+v k_{x}\right)^{3}=0,
\end{aligned}
$$

i.e. the same dispersion relation as in absence of optical dispersion except for the cubic term $\propto B_{0}$. We shall consider in what follows the $2 \mathrm{D}$ reduction, where $k_{\perp}=0$. Graphical solutions of the above equation are displayed in figure 2, Note that

$$
\omega_{l}=\gamma\left(\omega+v k_{x}\right),
$$

and then positive frequencies in the lab correspond to the region above the dashed line

$$
\omega=-v k_{x} .
$$

\section{B. Dispersion and horizons}

The definition of horizon which is suitable for this kind of situation, where one cannot recover a metric by reinterpreting the dispersion relation as an eikonal approximation of the wave equation (due to the presence of more 
than quadratic terms), can a priori involve phase velocity and/or group velocity; cf. e.g. a short discussion in [4]. Let us first consider what happens when one involves the phase velocity, indicated as $v_{l, \varphi}$ in the lab. reference frame and as $v_{\varphi}$ in the comoving frame:

$$
v_{l, \varphi}=v \Leftrightarrow v_{\varphi}=0
$$

In the lab. frame the calculation is elementary, and leads to

$$
v_{l, \varphi}= \pm \frac{c}{n\left(\omega_{l}, u\right)},
$$

which, due to 99 , implies the horizon condition

$$
n\left(\omega_{l}, u\right)=\frac{c}{v}
$$

it is worth noticing that condition 101 is a straightforward generalization to the dispersive case of the condition obtained in the absence of dispersion. It is also important to point out that the horizon condition in the lab in presence of dispersion can be also expressed as follows:

$$
1-n\left(\omega_{l}, u\right) \frac{v}{c}=0
$$

which e.g. in the Gaussian model provides

$$
u_{ \pm}= \pm \frac{\sigma}{\gamma} \sqrt{-2 \log \left(\left(\frac{c}{v}-n_{0}\left(\omega_{l}\right)\right) \frac{1}{\eta}\right)}
$$

and, in general, a modified horizon condition appears:

$$
n_{0}\left(\omega_{l}\right)<\frac{c}{v} \leq n_{0}\left(\omega_{l}\right)+\eta
$$

This condition is displayed in Fig. 3 (where wavelength replaces $\omega_{l}$ ) and the velocity of the RIP is chosen to match the experimental conditions of Ref. [26]. Explicitly, by using the Cauchy formula (87), we obtain (we consider only positive frequencies) for $n_{0}+\eta<\frac{c}{v}$, i.e. for a relatively small perturbation (see e.g. [26])

$$
\frac{\sqrt{\frac{c}{v}-n_{0}-\eta}}{\sqrt{B_{0}}} \leq \omega_{l}<\frac{\sqrt{\frac{c}{v}-n_{0}}}{\sqrt{B_{0}}}
$$

i.e., in terms of wavelength,

$$
\frac{2 \pi c \sqrt{B_{0}}}{\sqrt{\frac{c}{v}-n_{0}}}<\lambda \leq \frac{2 \pi c \sqrt{B_{0}}}{\sqrt{\frac{c}{v}-n_{0}-\eta}} .
$$

If, instead, $n_{0}+\eta \geq \frac{c}{v}$, i.e. for a substantially larger perturbation, we obtain

$$
0 \leq \omega_{l}<\frac{\sqrt{\frac{c}{v}-n_{0}}}{\sqrt{B_{0}}},
$$

i.e., in terms of wavelength,

$$
\frac{2 \pi c \sqrt{B_{0}}}{\sqrt{\frac{c}{v}-n_{0}}}<\lambda<\infty
$$

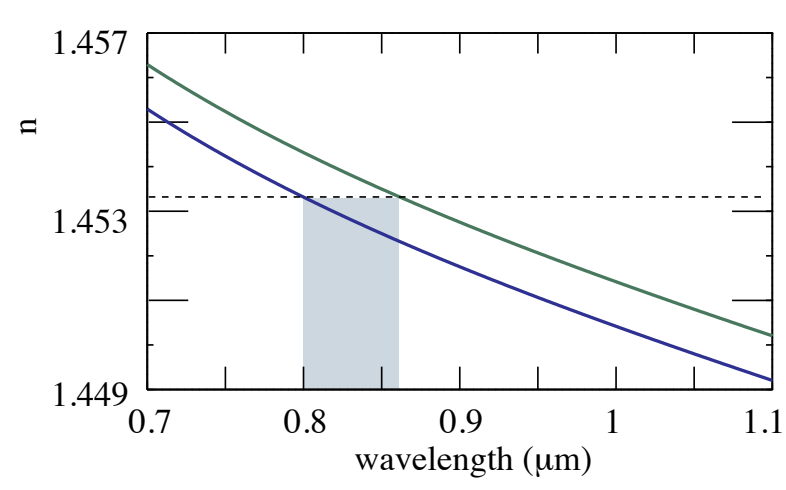

FIG. 3: (in color online) Prediction of the Hawking emission spectral range accounting for the RIP velocity and the material refractive index $n$. The two curves show, for the case of fused silica, $n_{0}$ (in blue) and $n_{0}+\delta n$ (in green) with $\delta n=1 \times 10^{-3}$. The shaded area indicates the spectral emission region predicted for a Bessel filament that has an effective refractive index $n=c / v_{B}$ where the Bessel peak velocity is $v_{B}=v_{G} / \cos \theta$ with cone angle $\theta=7 \mathrm{deg}$ and $v_{G}=d \omega / d k$ is the usual group velocity.

For the parameters of Fig. 3 (see caption), Eq. 107) is verified for $\eta>0.013$, a rather large yet not completely unrealistic refractive index variation. We may evaluate the influence of material dispersion in relation to the event horizon condition. In the absence of dispersion a horizon is created only if $v$ is tuned with extreme care such that Eq. 104 with dispersionless $n_{0}$ is satisfied. Bearing in mind the typically small values of $\delta n \sim 10^{-3}-10^{-4}$ (e.g. in fused silica, $n_{2} \sim 3 \cdot 10^{-16}$ $\mathrm{cm}^{2} / \mathrm{W}$ and $I \sim 10^{13} \mathrm{~W} / \mathrm{cm}^{2}$ ), this would be no minor feat.

Note that the Hawking photons will therefore in general be emitted only in a bounded spectral window. This is somewhat different from the dispersion-less case in which, once $v$ is properly tuned so as to achieve the horizon condition, all frequencies are simultaneously excited, and, a posteriori, it can also justify why one can limit oneself to adopt the Cauchy formula instead of a more complete Sellmeier. The aforementioned finite spectral window for photon emission in turn implies that in the dispersion-less case one should expect to observe the complete black-body spectrum predicted by Hawking. Conversely, in the presence of dispersion in our analogue model, only a limited spectral region is excited and the black-body spectral shape will not be discernible. Moreover, one has to take into account that, even in homogeneous transparent dielectrics, optical dispersion affects the spectral density of photons which is actually to be taken into account, by introducing a phase space factor multiplying the standard Planckian distribution term which depends on both the refractive index $n(\omega)$ and on 
the group velocity $v_{g}(\omega)$ 47]:

$$
\rho(\omega)=\frac{\left(\frac{\hbar \omega^{3}}{\pi^{2} c^{2}}\right) \frac{n^{2}(\omega)}{v_{g}(\omega)}}{\exp \left(\frac{\hbar \omega}{k_{b} T}\right)-1} .
$$

This formula is intended to hold true in the reference of the thermal bath. These optical dispersion contributions amounts to a sort of greybody factor arising from the interaction of photons with the dielectric material. See e.g. [47. This is per se a sufficient reason for expecting deviations from the standard Planckian distribution. A very naive inclusion of dispersion in our case would also lead to $T=T(\omega)$, which would make even less plausible a pure Planckian spectrum.

Regarding the width of the spectral emission window we note that this is determined by the value of $\eta$. For a typical case we consider a Gaussian pump pulse in fused silica, $n_{g}(800 \mathrm{~nm})=1.467$ and we find that for $\delta n=$ $10^{-3}$, Eq. 104 is satisfied over a $\sim 15 \mathrm{~nm}$ bandwidth at $435 \mathrm{~nm}$. This window may become substantially large in lower dispersion regions as shown in Fig. 3 .

The phase velocity horizon condition may also be calculated in the comoving frame, where one obtains $v_{\varphi}=0$ if $\omega=0$ (for $k_{x} \neq 0$ ), i.e. for $v>0$

$$
\left.n\left(\gamma\left(\omega+v k_{x}\right), x\right)\right|_{\left\{\omega\left(k_{x}, x\right)=0, k_{x} \neq 0\right\}}=\frac{c}{v} .
$$

Notice that $\omega=\omega\left(k_{x}, x\right)$ is solution of the $2 \mathrm{D}$ reduction of eqn. (96). The above condition requires that, as in absence of dispersion, $n_{0}<\frac{c}{v}$. Moreover, if also $n_{0}+\eta<$ $\frac{c}{v}$, then two disconnected regions are obtained:

$$
\begin{aligned}
&-\frac{\sqrt{\frac{c}{v}-n_{0}}}{\gamma v \sqrt{B_{0}}}<k_{x} \leq-\frac{\sqrt{\frac{c}{v}-n_{0}-\eta}}{\gamma v \sqrt{B_{0}}} \\
& \frac{\sqrt{\frac{c}{v}-n_{0}-\eta}}{\gamma v \sqrt{B_{0}}} \leq k_{x}<\frac{\sqrt{\frac{c}{v}-n_{0}}}{\gamma v \sqrt{B_{0}}},
\end{aligned}
$$

where only the latter region corresponds to $\omega_{l}>0$. These are shown in Fig. 4. Of course, the latter condition is equivalent to condition 105 in the lab frame.

If, instead, $n_{0}+\eta \geq \frac{c}{v}$, then a unique connected region is obtained, with

$$
-\frac{\sqrt{\frac{c}{v}-n_{0}}}{\gamma v \sqrt{B_{0}}}<k_{x}<\frac{\sqrt{\frac{c}{v}-n_{0}}}{\gamma v \sqrt{B_{0}}} .
$$

This condition is consistent with (107). For a further comment, see below.

One could also a priori consider a different definition of horizon, involving the group velocity instead of the phase velocity:

$$
v_{l, g}=v \Leftrightarrow v_{g}=0,
$$

where the first equality holds in the lab and the second in the pulse frame. For simplicity we consider a $2 \mathrm{D}$ reduction of our model, so that $k_{\perp}=0$. One obtains

$$
v_{l, g}=v \Leftrightarrow \frac{c}{v}=n\left(\omega_{l}, u\right)+\frac{\partial n\left(\omega_{l}, u\right)}{\partial \omega_{l}} \omega_{l} .
$$

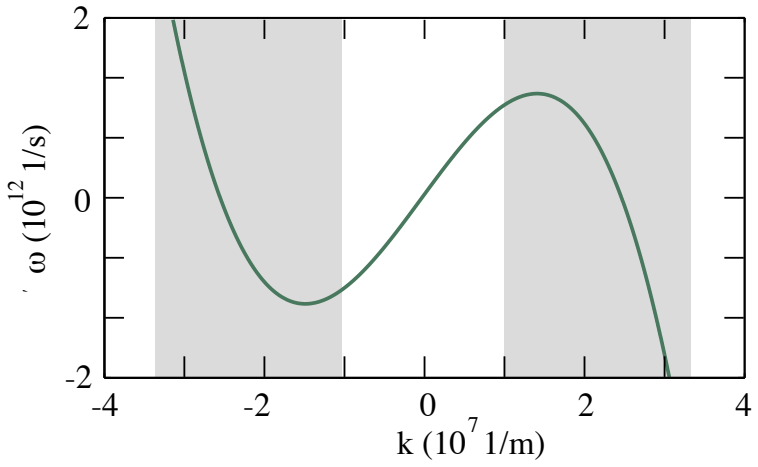

FIG. 4: (in color online) The dispersion relation (96) in the comoving frame for a $2 \mathrm{D}$ model at a fixed point $x$ (only the branch relevant to horizon formation is shown). The shaded regions delimit the bands representing the values of $k_{x}$ for which a phase horizon exists. See Eqs. (110-111).

In the case of the Cauchy formula, we find

$$
\frac{c}{v}=n\left(\omega_{l}, u\right)+2 B_{0} \omega_{l}^{2} \equiv n_{g}\left(\omega_{l}, u\right),
$$

where $n_{g}$ indicates the group refractive index. In this case, in place of (104), one would obtain

$$
n_{g}\left(\omega_{l}\right)<\frac{c}{v} \leq n_{g}\left(\omega_{l}\right)+\eta,
$$

which presents a clear shift with respect to the window corresponding to (104). Explicitly, by referring again to (87), we find

$$
\frac{\sqrt{\frac{c}{v}-n_{0}-\eta}}{\sqrt{3 B_{0}}} \leq \omega_{l}<\frac{\sqrt{\frac{c}{v}-n_{0}}}{\sqrt{3 B_{0}}},
$$

i.e., in terms of wavelength,

$$
\frac{2 \pi c \sqrt{3 B_{0}}}{\sqrt{\frac{c}{v}-n_{0}}}<\lambda \leq \frac{2 \pi c \sqrt{3 B_{0}}}{\sqrt{\frac{c}{v}-n_{0}-\eta}} .
$$

It is easy to realize that coexistence of phase and group horizons is allowed only if

$$
\frac{\sqrt{\frac{c}{v}-n_{0}}}{\sqrt{3 B_{0}}} \geq \frac{\sqrt{\frac{c}{v}-n_{0}-\eta}}{\sqrt{B_{0}}},
$$

i.e. if

$$
\eta \geq \frac{2}{3}\left(\frac{c}{v}-n_{0}\right) .
$$

Note that experiments, at least if the latter condition is not satisfied, should be able to distinguish between the two horizon conditions for phase velocity and group velocity given above. For example, in the experimental conditions of Ref. 26] used also in Fig. 3. condition (120) implies $\eta>0.009$. However the maximum $\eta$ obtained was only $\sim 0.001$ and indeed the experiments clearly reveal emission in correspondence to the phase horizon alone. 
It is possible to show that, if $\eta>\frac{c}{v}-n_{0}$, there is a region in the $\left(\omega, k_{x}, x\right)$-space where no group horizon appears because of the lack of any real zero in the derivative of the dispersion equation. In order to realize this fact analytically, we can proceed as follows. Let us consider in the comoving frame the dispersion equation in the following form:

$$
D=D_{+} D_{-}=0
$$

where

$D_{ \pm}:=n(x) \gamma\left(\omega+v k_{x}\right)+B_{0} \gamma^{3}\left(\omega+v k_{x}\right)^{3} \pm \gamma\left(k_{x}+\frac{v}{c^{2}} \omega\right) c=0$.

The dispersion relation $\omega\left(k_{x}, x\right)$ solves $D=0$. The only dispersion equation which can lead to a group velocity horizon is the solution $\omega_{-}\left(k_{x}, x\right)$ of $D_{-}=0$. Instead, the other branch $\omega_{+}\left(k_{x}, x\right)$ which solves $D_{+}=0$ is monotonically decreasing. We find

$$
v_{g}=\frac{\partial \omega\left(k_{x}, x\right)}{\partial k_{x}}=\frac{\partial D}{\partial k_{x}}\left(\frac{\partial D}{\partial \omega}\right)^{-1}
$$

As a consequence, a group velocity horizon can emerge only by solving the system $D_{-}=0$ and $\frac{\partial D_{-}}{\partial k_{x}}=0$. It is easy to show that $\frac{\partial D_{-}}{\partial k_{x}}=0$ leads to

$$
k_{x}^{(-)}=-\frac{\omega}{v} \pm \frac{1}{\gamma v \sqrt{3 B_{0}}} \sqrt{\frac{c}{v}-n(x)} .
$$

It is immediate to see that, if $n_{0}+\eta \geq \frac{c}{v}$, as in the case where a unique connected region is found for the phase velocity horizons (cf. eqn. 112), $k_{x}^{(-)}$in 124) is complex valued for all $x$ such that $n(x)>\frac{c}{v}$, for which then group velocity horizons disappear.

We may also qualitatively describe the behavior of a monochromatic or quasi-monochromatic wave near a phase horizon. In the comoving frame, an horizon is approached only by waves that travel with $v_{\varphi}>0$. Let us consider a wave traveling towards the white hole horizon; as it reaches the RIP, the refractive index increases, and then it slows down. This effect is enhanced by dispersion, because it implies a further increase of the refractive index. As the wave is as near as possible to the phase (white hole) horizon, it nearly stops, but, as remarked by e.g. [36], it cannot stop indefinitely, but, rather suffers reflection (mode conversion). This happens for all frequencies belonging to the allowed windows indicated above. The previous analysis is corroborated by the study of null geodesics, in the presence of optical dispersion, which is carried out in 49 . Indeed, therein it is shown (in a more general geometric setting) that only geodesics with $v_{\varphi} \geq 0$ undergo a slowing down process but, instead of suffering a process of trapping, as happens in absence of optical dispersion, they are reflected away from the trapping point.

In the case of a wave packet, the dynamics appear to be more complex, but, due to its being a superposition of monochromatic components, we can infer that what happens should be a sort of 'remastering' of the wave packet by the horizon in the following sense: frequencies beyond the allowed window are not affected by the presence of the horizon, whereas frequencies in the aforementioned window are selectively 'bounced back' by a mode conversion mechanism. In this sense, we can appreciate the peculiar behavior of the phase horizon as a sort of semipermeable membrane, or, even better, as a sort of mirror with a selective reflection bandwidth, which transmits only the frequencies not belonging to the given windows. The reflected frequencies will superimpose maybe even in the form of a wave packet. What is expected is that, in general, an incoming wave packet is converted into a reflected and possibly very broad and irregular wave train.

The action of a group velocity horizon appears to be different, in the sense that, although the existence of a window of allowed frequencies seems to be analogous to the one of a phase velocity horizon, we have to point out that in the present case $\omega_{l}$ refers to the carrier wave in the wave train. The group velocity horizon appears to be less selective than the phase velocity one: it limits itself to cause a reflection of wave packets which have carrier frequencies in the allowed window, without distinguishing between monochromatic components composing the packet itself. The outgoing wave train is expected to be still in the form of a compact and relatively undisturbed wave packet. Both numerical simulations and experimental results could be able to reveal this different character of the two above horizon versions, and in particular measurements should be able to discern which definition is relevant for the physics at hand. It is worth pointing out that, in Ref. [26], the main role in photon production appears to be related to the phase velocity horizon rather than to the group velocity horizon.

\section{CONCLUSIONS}

We have studied the photon production induced in a dielectric medium by a refractive index perturbation, which is created in a nonlinear medium by a laser pulse through the Kerr effect. The pulse has been assumed to have constant velocity $v$. In the pulse frame, we have investigated the analogous metric, and we have shown that two Killing horizons appear (black hole and white hole) for a generic but static dielectric perturbation. Despite the fact that the analogue metric is determined up to an overall conformal factor, the temperature is conformally invariant. Then we have taken into account quantum field theory in the lab frame, in a way that is independent of the analogous geometric framework we have again shown that there is a photon production with a thermal spectrum, corresponding to the Hawking effect. These findings are summarized in Table I. We have also provided the transformation law of the temperature between the given frames and taken into account the effects of dispersion on the horizon condition. 


\section{Acknowledgments}

F.Belgiorno wishes to thank Sergio Serapioni and Lesaffre Italia S.p.A. for financial support to the Department of Mathematics of the Università degli Studi di Milano, where part of this work was performed.

\section{Appendix A: Formal mapping to an acoustic black hole}

It is interesting to notice that our black hole metric in the pulse frame can be mapped into a form of acoustic black hole metric, provided that suitable identifications are made. In particular, we refer to the acoustic black hole metrics taken into consideration in [50], and limit ourselves to the analysis of the $x-t$-part of the metric (indeed, only 2D metrics are studied in [50]). In particular, we are looking for a transformation allowing to carry

$$
\begin{aligned}
d s_{(2)}^{2} & =-\frac{\gamma^{2}}{n^{2}}\left[-\left(c^{2}-n^{2} v^{2}\right) d t^{2}\right. \\
& \left.-2 v\left(1-n^{2}\right) d t d x+\left(n^{2}-\frac{v^{2}}{c^{2}}\right) d x^{2}\right]
\end{aligned}
$$

into the form

$$
d s_{\text {acoustic }}^{2}=-\Omega^{2}\left[-\left(\tilde{c}^{2}-\tilde{v}^{2}\right) d t^{2}-2 \tilde{v} d t d \tilde{x}+d \tilde{x}^{2}\right],
$$

where $\tilde{v}$ plays the role of fluid velocity (in general depending on $t, \tilde{x}$ ) and $\tilde{c}$ is the local speed of sound, assumed to be constant as in [50. This re-mapping of the optical metric into an acoustic one is implemented by the identifications

$$
\begin{aligned}
\tilde{c} & =c, \\
\tilde{v} & =\gamma^{2} v \frac{n^{2}-1}{n}, \\
\Omega^{2} & =\frac{1}{\gamma^{2}} \frac{1}{n^{2}-\frac{v^{2}}{c^{2}}}, \\
\frac{d \tilde{x}}{d x} & =-\gamma^{2} \frac{n^{2}-\frac{v^{2}}{c^{2}}}{n} .
\end{aligned}
$$

It is straightforward to check that the horizon condition $\tilde{v}=c$ is equivalent to the condition $n=\frac{c}{v}$, i.e. the solution of (9). By defining

$$
\begin{aligned}
& d \tilde{u}=d t-\frac{d \tilde{x}}{\tilde{c}+\tilde{v}}, \\
& d \tilde{w}=d t+\frac{d \tilde{x}}{\tilde{c}-\tilde{v}},
\end{aligned}
$$

as in 50, one can also easily draw the Penrose diagram of our spacetime (see Fig. 5), which is analogous to that

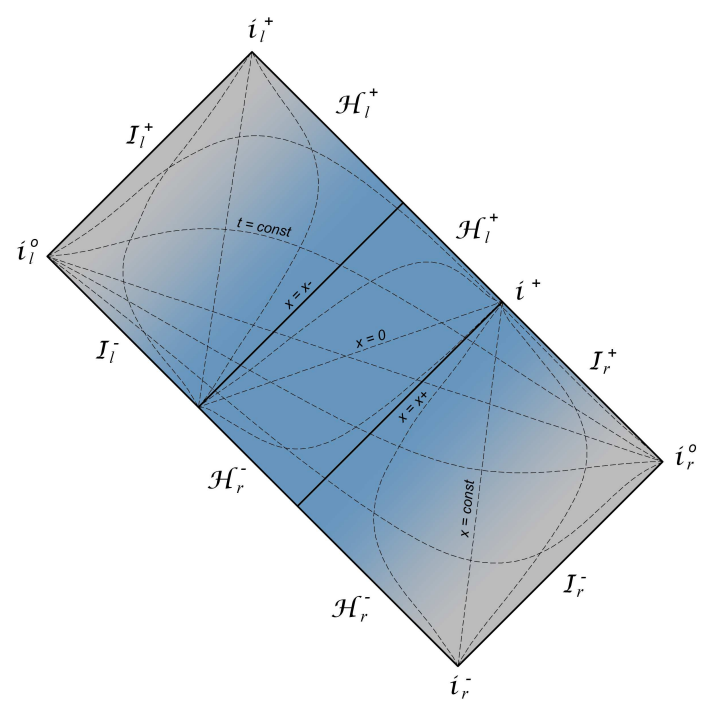

FIG. 5: Penrose diagram for the analogue metric (2).

of [50, Fig. 28]. It is also straightforward to verify that the surface gravity can be calculated also by means of the formula

$$
\kappa_{+}=\tilde{c}\left|\frac{\partial}{\partial \tilde{x}}(\tilde{c}-\tilde{v})\right|_{x=x_{+}}
$$

(cf. [32]).

\section{Appendix B: the Hawking temperature and its conformal invariance}

We consider the Euclidean version of the metric and find that near the horizon the $c t-x$ part of the metric behaves like a cone which becomes a flat plane only if a special choice of an angle parameter, to be related to the inverse of the Hawking temperature, is chosen.

The following rescaling to dimensionless variables is performed:

$$
\begin{aligned}
c \tau \in(0, \beta) & \mapsto \psi=\frac{2 \pi}{\beta} c \tau \in(0,2 \pi) \\
x \in\left(x_{+}, \infty\right) & \mapsto \bar{x}=\frac{2 \pi}{\beta} x \in\left(\frac{2 \pi}{\beta} x_{+}, \infty\right),
\end{aligned}
$$

where $\tau$ stays for the Euclidean time. Moreover, the following (local) diffeomorphism on the $c t-x$ part of the metric is introduced:

$$
\begin{aligned}
d s_{(2)}^{2} & =-\left(\frac{\beta}{2 \pi}\right)^{2}\left[\frac{1}{n^{2}(\bar{x})} g_{\tau \tau}(\bar{x}) d\left(c^{2} \tau^{2}\right)+\frac{1}{g_{\tau \tau}(\bar{x})} d \bar{x}^{2}\right] \\
& =-\left(\frac{\beta}{2 \pi}\right)^{2} H^{2}(y)\left(y^{2} d \psi^{2}+d y^{2}\right),
\end{aligned}
$$


whence

$$
\begin{aligned}
\frac{1}{n^{2}(\bar{x})} g_{\tau \tau}(\bar{x}) & =y^{2} H^{2}(y), \\
\frac{1}{g_{\tau \tau}(\bar{x})}\left(\frac{d \bar{x}}{d y}\right)^{2} & =H^{2}(y) .
\end{aligned}
$$

The above diffeomorphism is such that the $c t-x$ part of the metric near the horizon can, at least locally, be made conformal to a 2D plane with a suitable choice of $\beta$, with conformal factor $\left(\frac{\beta}{2 \pi}\right)^{2} H^{2}(y)$. As a consequence, one finds (taking the positive sign)

$$
\frac{2 \pi}{\beta} \frac{d x}{d y}=\frac{1}{y} \frac{1}{n(x)} g_{\tau \tau}(x),
$$

i.e.

$$
y=A \exp \left(\frac{2 \pi}{\beta} \int^{x} d z n(z) \frac{1}{g_{\tau \tau}(z)}\right),
$$

where $A$ is an integration constant. The diffeomorphism is defined to be regular if we can include in the manifold also the point $y=0$, and then we have to require

$$
\lim _{y \rightarrow 0^{+}} H^{2}(y)=\lim _{y \rightarrow 0^{+}} \frac{1}{y^{2}} g_{\tau \tau}(x(y))=h_{0}^{2},
$$

where $h_{0}^{2}$ is a finite positive constant. The above limit is equivalent to

$$
\lim _{x \rightarrow x_{+}} \frac{1}{y^{2}(x)} g_{\tau \tau}(x)
$$

Near the horizon one finds

$$
y^{2} \sim\left(x-x_{+}\right)^{\frac{2 \pi}{\beta} \frac{c^{2}}{v^{2}} \frac{1}{\gamma^{2}} \frac{1}{-\frac{d n}{d x}\left(x_{+}\right)}}
$$

and, as a consequence, one has to choose

$$
\beta=2 \pi \frac{c^{2}}{v^{2}} \frac{1}{\gamma^{2}} \frac{1}{-\frac{d n}{d x}\left(x_{+}\right)}=: \hbar c \beta_{h} .
$$

The above method can be used also to confirm that the temperature does not depend on the (static) conformal factor. Indeed, an overall conformal factor $\Omega^{2}(x)$ which is finite and non-vanishing at the horizons ( $\lim _{x \rightarrow x_{ \pm}} \Omega^{2}(x)=b_{ \pm}^{2}>0$ ) does not modify Eq. (B10, as may be realized using Eqs. (B8) and (B9).

\section{Appendix C: Exact analysis of equation (26) near the singularity}

We confine ourselves to discuss the root $u_{+}$, since all considerations can be trivially extended to $u_{-}$. Then, we rewrite Eq. 26) as

$$
A^{\prime \prime}(u)+\frac{P(u)}{u-u_{+}} A^{\prime}(u)+\frac{Q(u)}{u-u_{+}} A(u)=0,
$$

where we have introduced the functions $P(u)$ and $Q(u)$ (holomorphic in the disk $\left|u-u_{+}\right|<\left|u_{+}-u_{-}\right|$)

$$
\begin{gathered}
P(u)=2 i k_{w} \frac{c^{2}+n^{2}(u) v^{2}}{c^{2}-n^{2}(u) v^{2}}, \\
Q(u)=-\left(k_{w}^{2}+\frac{k_{y}^{2}+k_{z}^{2}}{1-n^{2}(u) \frac{v^{2}}{c^{2}}}\right) .
\end{gathered}
$$

In a neighbourhood of $u_{+}$Eq. 26) writes, to leading order in $u-u_{+}$, as

$$
\begin{aligned}
A^{\prime \prime}(u)-\frac{2 i k_{w} c}{v n^{\prime}\left(u_{+}\right)} & \frac{1}{u-u_{+}} A^{\prime}(u)+ \\
& +\frac{\left(k_{y}^{2}+k_{z}^{2}\right) c}{2 v n^{\prime}\left(u_{+}\right)\left(u-u_{+}\right)} A(u)=0,
\end{aligned}
$$

where the prime denotes derivation with respect to the lab variable $u$. The indicial equation is $\alpha(\alpha-1)-$ $\alpha \frac{2 i k_{w} c}{v n^{\prime}\left(u_{+}\right)}=0$, with roots $\alpha_{1}=0$ and $\alpha_{2}=1+\frac{2 i k_{w} c}{v n^{\prime}\left(u_{+}\right)}$. Therefore, in the neighbourhood of $u_{+}$, Eq. (26) has two linearly independent solutions of the form

$$
A^{(i)}(u)=\left(u-u_{+}\right)^{\alpha_{i}} \sum_{n=0}^{\infty} c_{n}^{(i)}\left(u-u_{+}\right)^{n},
$$

$i=1,2$, where the series define holomorphic functions in the disc $\left|u-u_{+}\right|<\left|u_{+}-u_{-}\right|$and whose coefficients can be obtained recursively from the equation $c_{n}^{(i)}\left(\alpha_{i}+\right.$ $n)\left(\alpha_{i}+n-1+p_{0}\right)+\sum_{r=0}^{n-1}\left(\left(\alpha_{i}+r\right) p_{n-r}+q_{n-r-1}\right) c_{r}^{(i)}=0$, where the $p_{k}, u_{k}(k=0,1,2, \ldots)$ are the coefficients of the expansion about $u_{+}$of $P(u)$ and $Q(u)$ respectively. Of particular interest is the solution (25) corresponding to $\alpha=\alpha_{2}$, which for $u>u_{+}$has the form

$$
F_{\alpha_{2}}(u, w, y, z)=\xi(u) \mathrm{e}^{i \frac{2 k_{w} c}{v n^{\prime}(u+)} \log \left(u-u_{+}\right)+i k_{w} w+i k_{y} y+i k_{z} z},
$$

where $\xi(u)$ is holomorphic in the neighbourhood of $u=$ $u_{+}$and vanishes as $u \rightarrow u_{+}$: indeed, we have reabsorbed in it the factor $\left(u-u_{+}\right)$associated with the real term in $\alpha_{2}$, in such a way that

$$
\xi(u)=\left(u-u_{+}\right) \eta(u),
$$

where $\eta(u)$ is holomorphic in the neighbourhood of $u=$ $u_{+}$and $\eta(u)=c_{0}+c_{1}\left(u-u_{+}\right)+\ldots$ as $u \rightarrow u_{+}$. We stress the presence of the logarithmic divergence of the phase as $u$ approaches $u_{+}$even in this approach. Also, by comparison with the study of the solutions contained in section III A, we can infer that the above solution corresponds to the (exact) expansion of $F_{\mathbf{k}}^{+}(u, w, y, z)$ near $u=u_{+}$.

\section{Appendix D: Analytic continuations}

We are interested in the Bogoliubov coefficient $\alpha_{\mathbf{k k}^{\prime}}$, which relates the positive frequencies between the initial 
in state:

$$
F_{\mathbf{k}}^{\text {in }}=e^{i k_{x} x+i k_{y} y+i k_{z} z-i \omega t},
$$

and final out state $F_{\mathbf{k}}^{+}$:

$$
F_{\mathbf{k}}^{+}=\theta\left(u-u_{+}\right) \xi_{\mathbf{k}}(u) e^{i\left(\sigma \log \left(u-u_{+}\right)+k_{w} w+k_{y} y+k_{z} z\right)},
$$

where we have introduced the shorthand notation $\sigma=$ $\frac{2 c}{n^{\prime}\left(u_{+}\right) v} k_{w}$ and, in agreement with the results obtained in Appendix C, we have introduced also the analytic part $\xi(u)$. It will be evident that, at least in the large frequency limit, this $\xi(u)$ cannot affect the thermal character of particle emission.

Let us start with the computation of $\alpha_{\mathbf{k k}^{\prime}}$, which, apart from a factor which will not affect our goal, which consists in the deduction of (D7), is given by

$$
\alpha_{\mathbf{k k}^{\prime}} \propto \delta^{2}\left(\mathbf{k}_{\perp}-\mathbf{k}_{\perp}^{\prime}\right) \int_{0}^{\infty} \xi(u) u^{i \sigma} e^{-i k_{u}^{\prime} u} d u,
$$

where we have shifted the variable $u$ so that $u_{+}$is mapped on 0 . Since $2 k_{u}^{\prime}=\left(k_{x}^{\prime}+\omega^{\prime} / v\right)>0$, we see that the second exponential factor is rapidly decreasing at infinity when $\operatorname{Im}(u)<0$. Consider the path $\Gamma$ starting from 0 to $R>0$ along the real line, then following the arc of radius $R$ clockwise until $-i R$, and finally coming back from $-i R$ to 0 along the imaginary axis. As the integrand is analytic inside the region bounded by the path, the integral along $\Gamma$ vanishes for any positive value of $R$. Then, taking the limit $R \rightarrow+\infty$ we then see that the integral along the positive real axis is equal to the integral along the negative imaginary axis and

$$
\alpha_{\mathbf{k k}^{\prime}} \propto e^{\frac{\pi}{2} \sigma} \frac{\delta^{2}\left(\mathbf{k}_{\perp}-\mathbf{k}_{\perp}^{\prime}\right)}{i\left(k_{u}^{\prime}\right)^{1+i \sigma}} \int_{0}^{\infty} d t \xi\left(\frac{-i t}{k_{u}^{\prime}}\right) t^{i \sigma} e^{-t} .
$$

We can consider the limit as $k_{u}^{\prime} \gg 1$ and approximate $\xi$ for small values of its argument. Then we obtain

$$
\alpha_{\mathbf{k k}^{\prime}} \propto e^{\frac{\pi}{2} \sigma} \frac{\delta^{2}\left(\mathbf{k}_{\perp}-\mathbf{k}_{\perp}^{\prime}\right)}{\left(k_{u}^{\prime}\right)^{2+i \sigma}} \Gamma(2+i \sigma)
$$

where $\Gamma$ is the Euler Gamma function.

On the other hand, in order to calculate $\beta_{\mathbf{k k}^{\prime}}$, it is sufficient to revert the sign of $k_{u}^{\prime}$ and $\mathbf{k}^{\prime}$ in Eq. (D3). This time, the integral can be calculated along the positive imaginary axis, since now the term relative to the integration on the arc vanishes for $\operatorname{Im}(u)>0$. Thus, rotating the path counter-clockwise, we obtain

$$
\beta_{\mathbf{k k}^{\prime}} \propto e^{-\frac{\pi}{2} \sigma} \frac{\delta^{2}\left(\mathbf{k}_{\perp}+\mathbf{k}_{\perp}^{\prime}\right)}{\left(k_{u}^{\prime}\right)^{2+i \sigma}} \Gamma(2+i \sigma),
$$

By comparing Eqs. (D5) and (D6), it is easy to verify that

$$
\sum_{\mathbf{k}^{\prime}}\left|\alpha_{\mathbf{k} \mathbf{k}^{\prime}}\right|^{2}=e^{2 \pi \sigma} \sum_{\mathbf{k}^{\prime}}\left|\beta_{\mathbf{k} \mathbf{k}^{\prime}}\right|^{2}
$$

which is Eq. 70 .
[1] S.W. Hawking, Nature 248, 30 (1974).

[2] S.W. Hawking, Commun. Math. Phys. 43, 199 (1975).

[3] C. Kiefer, in Classical and Quantum Black Holes, P. Fré, V. Gorini, G. Magli and U. Moschella Eds. IOP Publishing, Bristol (1999).

[4] M. Visser, Phys. Rev. Lett. 80, 3436 (1998).

[5] M. Visser, Int. J. Mod. Phys. D12, 649 (2003).

[6] C. Barcelo, S. Liberati, S. Sonego and M. Visser, Phys. Rev. Lett. 97, 171301 (2006).

[7] W.G. Unruh, Phys. Rev. Lett. 46, 1351 (1981).

[8] C. Barcelò, S. Liberati, and M. Visser, Living Rev. Relativity $\mathbf{8 ,} 12$ (2005).

[9] J. Macher and R. Parentani, Phys. Rev. A 80, 043601 (2009).

[10] W. Gordon, Ann. Phys. (Leipzig) 72, 421 (1923).

[11] U. Leonhardt and P. Piwnicki, Phys. Rev. A 60, 4301 (1999).

[12] U. Leonhardt and P. Piwnicki, Phys. Rev. Lett. 84, 822 (2000).

[13] R. Schutzhold, G. Plunien and G. Soff, Phys. Rev. Lett. 88, 061101 (2002)

[14] I. H. Brevik and G. Halnes, Phys. Rev. D 65, 024005 (2002).

[15] V. A. De Lorenci and R. Klippert, Phys. Rev. D 65, 064027 (2002).

[16] V. A. De Lorenci and M. A. Souza, Phys. Lett. B 512,
417 (2001).

[17] M. Novello and J. M. Salim, Phys. Rev. D 63, 083511 (2001).

[18] M. Marklund, D. Anderson, F. Cattani, M. Lisak and L. Lundgren, Am. J. Phys. 70, 680 (2002).

[19] V. A. De Lorenci, R. Klippert and Yu. N. Obukhov, Phys. Rev. D 68, 061502 (2003).

[20] M. Novello, S. E. Perez Bergliaffa, J. Salim, V. De Lorenci and R. Klippert, Class. Quant. Grav. 20, 859 (2003).

[21] M. Novello and S. E. Perez Bergliaffa, AIP Conf. Proc. 668, 288 (2003).

[22] W. G. Unruh and R. Schutzhold, Phys. Rev. D 68, 024008 (2003).

[23] R. Schutzhold and W. G. Unruh, Phys. Rev. Lett. 95, 031301 (2005).

[24] T.G. Philbin, C. Kuklewicz, S. Robertson, S. Hill, F. Konig, and U. Leonhardt, Science 319, 1367 (2008).

[25] D. Faccio, S. Cacciatori, V. Gorini, V.G. Sala, A. Averchi, A. Lotti, M. Kolesik, and J.V. Moloney, EuroPhys. Lett., EPL. 89, 34004 (2010).

[26] F. Belgiorno, S.L. Cacciatori, M. Clerici, V. Gorini, G. Ortenzi, L. Rizzi, E. Rubino, V.G. Sala, and D. Faccio, "Hawking radiation from ultrashort laser pulse laments," Phys. Rev. Lett. 105, 203901 (2010).

[27] W.G. Unruh, Phys. Rev. D 14, 870 (1976).

[28] J. Schwinger, Proc. Natl. Acad. Sci. USA 89, 4091 
(1992); 8911118 (1992); 90, 958 (1993); 90, 2105 (1993); 90, 4505 (1993); 90, 7285 (1993); 91, 6473 (1994).

[29] D. Faccio, F. Belgiorno, S. Cacciatori, M. Clerici, V. Gorini, G. Ortenzi, L. Rizzi, E. Rubino, V.G. Sala, "Analogue gravity and ultrashort laser pulse filamentation." Invited paper. In 'Nonlinear Optics and Applications IV', edited by Benjamin J. Eggleton, Alexander Luis Gaeta, Neil G. R. Broderick Proc. of SPIE Vol. 7728, 77280M (2010).

[30] R.M. Wald, General Relativity. The University of Chicago Press, Chicago (1984).

[31] T. Jacobson and G. Kang, Class. Quant. Grav. 10, L201 (1993).

[32] M. Visser, Class. Quant. Grav. 15, 1767 (1998).

[33] A. Coutant and R. Parentani, Phys. Rev. D 81, 084042 (2010).

[34] S.Flugge, Practical Quantum Mechanics. Springer, New York (1999).

[35] W. G. Unruh, Phys. Rev. D 51, 2827 (1995).

[36] S. Corley and T. Jacobson, Phys. Rev. D 54, 1568 (1996).

[37] T. Jacobson, Phys. Rev. D 53, 7082 (1996).

[38] J. Macher and R. Parentani, Phys. Rev. D 79, 124008 (2009).

[39] A. Recati, N. Pavloff and I. Carusotto, Phys. Rev. A 80, 043603 (2009).

[40] R. Balbinot, A. Fabbri, S. Fagnocchi, A. Recati and I. Carusotto, Phys. Rev. A 78, 021603 (2008).
[41] S. Corley and T. Jacobson, Phys. Rev. D 59, 124011 (1999).

[42] S. Finazzi and R. Parentani, "Black-hole lasers in BoseEinstein condensates." arXiv:1005.4024

[43] U. Leonhardt and T.G. Philbin, Black-hole lasers revisited, in Quantum Analogues: From Phase Transitions to Black Holes and Cosmology edited by W. G. Unruh and R. Schützhold. Springer, Berlin (2007).

[44] B. Huttner and S. M. Barnett, Phys. Rev. A 46, 4306 (1992); B. Huttner, J. J. Baumberg and S. M. Barnett, Europhys. Lett. 16, 177 (1991).

[45] W.G. Unruh and R. Schutzhold, Phys. Rev. D 68, 024008 (2003).

[46] L.G. Suttorp and A.J. van Wonderen, Europhys. Lett. 67, 766 (2004).

[47] P.W. Milonni, J. Mod. Opt. 42, 1991 (1995).

[48] S. Robertson and U. Leonhardt, Phys. Rev. A 81, 063835 (2010).

[49] S. L. Cacciatori, F. Belgiorno, V. Gorini, G. Ortenzi, L. Rizzi, V. G. Sala and D. Faccio, New J. Phys. 12, 095021 (2010).

[50] C. Barcelo, S. Liberati, S. Sonego and M. Visser, New J. Phys. 6, 186 (2004).

[51] the factor $c^{4}$ appears since we are using standard units in place of the customary natural ones 